\title{
Integral Command Filtered Backstepping Control of a Flexible UAV
}

\author{
Ding Han ${ }^{1}$, Lin Yan², Guozheng Yan ${ }^{1}$, Xiaoliang Wang ${ }^{3}$, Dengping Duan ${ }^{3}$ \\ ${ }^{1}$ School of Electronic Information and Electrical Engineering, Shanghai Jiao Tong University, China \\ ${ }^{2}$ Computer Science, University of Utah, America \\ ${ }^{3}$ School of Aeronautics and Astronautics, Shanghai Jiao Tong University, China
}

\begin{abstract}
Airships, as the significant UAV, have a need for greater autonomy in their new missions. Therefore, airship flight control systems require precise dynamic modeling, taking into account the effect of flexibility and the interaction with aerodynamic forces. This research effort develops an efficient modeling of the autonomous flexible airship. The formalisation used is based on the Lagrange method. The resulting model includes the rigid body motion, the elastic deformation, and the coupling between them. Based on the precise flexible dynamic model, a novel backstepping nonlinear controller with integral action is proposed for motion control systems. The resulting feedback controller is able to adapt to actuator performance limitations, such as limitations in magnitude and rate of change of rudder, than conventional backstepping controller without integral action. With the deformation considered, the presented controller could resist the flexible uncertainty effect, and the system's trajectory tracking ability is significantly improved. The approach guarantees exponential stability of a compensated tracking error in the sense of Lyapunov.
\end{abstract}

\section{Introduction}

Airships, as autonomous aerial vehicles, have recently gained importance. The range of tasks they are designed to support is growing. For example, climate research, data collecting, surveillance, and more closer to us, we always saw them as means of advertising [1]. The resurgence of airships in the past few years has created a need for accurate dynamics models and simulation tools to study their flight behavior and design their control systems. Several researchers have presented airship dynamic models. Tischler et al. proposed the nonlinear equations of motion and developed the simulation program HLASIM for the design of heavy lift airships [2]. Jex and Gelhausen and Amann used different aerodynamic computational methods to predict the control responses of the Skyship-500 airship [3, 4]. Analytical linear dynamics models using aerodynamic derivatives were also applied to study the flight stability and control design of airships [5, 6]. Li and Nahon presented a modeling procedure for airship dynamics simulation and validated their model with published experimental data [7]. All of these works were researched by using the assumption of rigid body behavior. Sometimes the flexibility effects are modeled as a perturbation. However, real airships experience elastic deformations and their structural flexibility has been an important consideration in the history of airship development. In other vehicles, such as light aircraft, the introduction of the flexibility in the dynamic model becomes essential $[8,9,10]$. Thus we try to contribute to the study of the elastic deformation of the airships by considering the effect of flexibility. To design the control system, the deformation of the airship is considered acting on the motion rather than as a perturbation.

Recently, with increasing interest in airships and a drive to make them more autonomous, there has been increased research effort on airship modelling and control. Airships have different nonlinearities from heavier-thanair aircraft, such as added mass terms that are negligible for other aircraft. The airship has been less extensively studied than that for other aircraft. Due to the flexible structures and the time-varying characters, obtaining the precise mathematical models valid for relevant flight conditions is more difficult. Traditionally, to overcome this problem, airship models are usually linearized, and the flight controllers are designed for a selected flight condition. Several control techniques are then applied, usually with excellent results [10]. However, if the state of the airship is too different from the flight condition used for design, the performance of controller is not as optimal as before, and the airship model might fail to accurately capture the system behavior. In this situation, linear control laws can be prone to misbehavior or even failure. Gain scheduling methods have been successfully developed to deal with different operating points [11]. However, the disadvantages of these methods are that the controllers are not global used and a wide range of flight conditions need to be estimated, which is a complicated task.

Nonlinear control methods have been developed to overcome the shortcomings of linear design approaches. 
For example, feedback linearization has been used to handle the nonlinear equations of motion, generating controllers suitable for the entire flight envelope, if a precise knowledge of the aircraft model exists [12]. For security reasons, as well as simplicity and flexibility, a global nonlinear control is more interesting than a linearized and decoupled one. Lyapunov function based on backstepping control is described in [13], and have been proposed in [14], [15], and [16]. In reference [14], Godhavn derives a full nonlinear model and allows large variation in velocities. He used splines to make references smoothly and backstepping control to track them [14]. In reference [15], the dynamical extension is proposed. Fierro and Lewis consider the specific vehicle dynamics to convert a steering system (kinematic model) command into control inputs for the actual vehicle [15]. Encarnacao and Pascoal provide a control method that combines both the trajectory tracking and path following problems and shows a good tracking and following performance [16]. In the presence of parametric uncertainties, an alternative solution is to design the controller using the adaptive backstepping design method. For example, Härkegård has presented an adaptive backstepping design in [17], complemented with a control allocation method to compute actuator values. However, these designs do not take into account input saturation and rate limitation of the actuators, which will constrain the efficiency of the designed control law, or even cause an accident.

In recent control systems, the overall performance of the motion control systems is restricted by the limitation of magnitude and rate of change of the actuators, such as rudder angles. What's more, the elastic deformation of airship during flight motion process is also an complicating factor for control systems. So it is of great importance to design advanced controllers to achieve the bandwidth improvement.

In this work, a command filtered backstepping control law with integral action is designed for airship flexible dynamics. The resulting control law is able to track the given yaw reference considering the limitations in magnitude and rate of change of actuators. The proposed control law shows the good performance when considering actuator position and rate limits.

The paper is organized as follows: Section 2 establishes the mathematical flexible model of the airship. Section 3 presents a detailed derivation of the command filtered backstepping control law with integral action and deformation considered to deal with airship dynamics. Some simulations are carried out to show the performance and stability of the proposed controller in section 4. Finally, in section 5, the paper is concluded with some observations.

\section{Dynamics modeling of flexible airships}

Generally, the hull of an airship is a flexible inflating structure. The shape of an airship hull will change under the influence of aerodynamics. So the changes in shape, aerodynamics and attribute parameters need to be considered in the dynamics modeling. The main differences of flexible dynamic modeling and the rigid dynamic modeling are the hull shape change model, the aerodynamic model, the added masses and attribute parameters model.

The aerodynamic force will be changed with the flow condition and the shape of flexible airship. For obtaining the aerodynamics under the conditions of different flow condition and flexible shape, we use the semi-experiential estimate method [18] in this paper. But the change of local relative velocity and shape along the $\mathrm{x}$ axis of airship hull was contained in the aerodynamic model. The coordinate of airship is shown in Fig. 1.

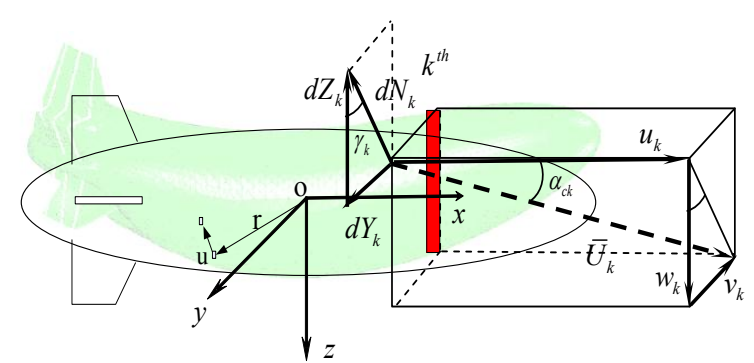

Figure 1. The body coordinate and parameters definition.

\subsection{The attribute parameters model}

The attribute parameters of an airship include the mass and inertia. Based on the structure and shape characteristics of airship, the airship was divided into different parts. The mass distribution of an airship includes the structure and interior gas. The hull was divided into $N_{g}$ panels. The interior gas was divided into $N_{x g}$ cylindrical segments. The fins and cabin were seen as concentrated mass parts. This method can take into account the mass distribution and change of shape of the airship in detail.

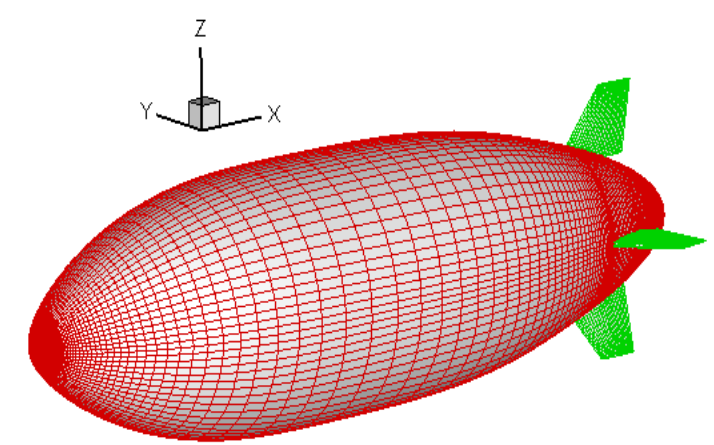

Figure 2. The discrete airship surface.

Thus, the coordinate of every panel is

$$
\mathbf{r}=\left[\begin{array}{l}
x(g) \\
y(g) \\
z(g)
\end{array}\right], \quad g=1,2, \cdots, N_{g}
$$

The mass of every panel is $\Delta m(g), \quad g=1,2, \cdots N_{g}$

In addition, every natural mode shape can be discretised, 


$$
\boldsymbol{\Phi}_{i}(\mathbf{r})=\left[\begin{array}{c}
\Phi_{x i}(\mathbf{r}) \\
\Phi_{y i}(\mathbf{r}) \\
\Phi_{z i}(\mathbf{r})
\end{array}\right]=\left[\begin{array}{c}
\Delta x_{i}(g) \\
\Delta y_{i}(g) \\
\Delta z_{i}(g)
\end{array}\right], \quad g=1,2, \cdots, N_{x g}
$$

\subsection{The flexible dynamic model of airship}

Through the dynamic equations of flexible body, the dynamic model of flexible airship can be obtained [19],

$$
\begin{aligned}
{\left[\begin{array}{lll}
\mathbf{M}_{11} & \mathbf{M}_{12} & \mathbf{M}_{13} \\
\mathbf{M}_{21} & \mathbf{M}_{22} & \mathbf{M}_{23} \\
\mathbf{M}_{31} & \mathbf{M}_{32} & \mathbf{M}_{33}
\end{array}\right]\left[\begin{array}{c}
\dot{\mathbf{v}}_{\mathbf{0}} \\
\mathbf{\mathbf { \Omega }} \\
\ddot{\mathbf{q}}
\end{array}\right]=} & -\left[\begin{array}{c}
\mathbf{0} \\
\mathbf{0} \\
\mathbf{S}_{\mathbf{E}}
\end{array}\right]+\left[\begin{array}{c}
\mathbf{F}_{\mathbf{I}} \\
\mathbf{M}_{\mathbf{I}} \\
\mathbf{Q}_{\mathbf{I}}
\end{array}\right]+\left[\begin{array}{c}
\mathbf{F}_{\mathbf{G}} \\
\mathbf{M}_{\mathbf{G}} \\
\mathbf{Q}_{\mathbf{G}}
\end{array}\right] \\
& +\left[\begin{array}{c}
\mathbf{F}_{\mathrm{AS}} \\
\mathbf{M}_{\mathrm{AS}} \\
\mathbf{Q}_{\mathrm{AS}}
\end{array}\right]+\left[\begin{array}{c}
\mathbf{F}_{\mathrm{AD}} \\
\mathbf{M}_{\mathbf{A D}} \\
\mathbf{Q}_{\mathrm{AD}}
\end{array}\right]+\left[\begin{array}{c}
\mathbf{F}_{\mathbf{C}} \\
\mathbf{M}_{\mathbf{C}} \\
\mathbf{Q}_{\mathbf{C}}
\end{array}\right]
\end{aligned}
$$

where, $\mathbf{M}_{s y s}$ is the symmetry mass matrix,

$$
\mathbf{M}_{s y s}=\left[\begin{array}{lll}
\mathbf{M}_{11} & \mathbf{M}_{12} & \mathbf{M}_{13} \\
\mathbf{M}_{21} & \mathbf{M}_{22} & \mathbf{M}_{23} \\
\mathbf{M}_{31} & \mathbf{M}_{32} & \mathbf{M}_{33}
\end{array}\right]
$$

$\mathbf{S}_{\mathbf{E}}$ is the interior elastic force vector, $\mathbf{S}_{\mathbf{E}}=\mathbf{K q}, \mathbf{K}$ is the stiffness matrix,

$$
K_{i, j}=K_{N+i, N+j}=\int_{L} E I \Psi_{i}^{\prime \prime} \Psi_{j}^{\prime \prime} d x, i, j=1,2 \cdots, N
$$

where $E I$ is the bend stiffness, $\Psi_{i}^{\prime \prime}=\frac{d^{2} \Psi_{i}}{d x^{2}}$.

The right term of the dynamic model of flexible airship is the exterior forces, $\mathbf{F}_{\mathbf{I}}, \mathbf{M}_{\mathbf{I}}, \mathbf{Q}_{\mathbf{I}}$ are the inertia force and moment; $\mathbf{F}_{G}, \mathbf{M}_{G}, \mathbf{Q}_{G}$ are the gravity and gravity moment; $\mathbf{F}_{\mathrm{AS}}, \mathbf{M}_{\mathrm{AS}}, \mathbf{Q}_{\mathrm{AS}}$ are the buoyancy and buoyancy moment; $\mathbf{F}_{\mathbf{A D}}, \mathbf{M}_{\mathrm{AD}}, \mathbf{Q}_{\mathrm{AD}}$ are the aerodynamic force and moment; $\mathbf{F}_{\mathbf{C}}, \mathbf{M}_{\mathbf{C}}, \mathbf{Q}_{\mathrm{C}}$ are the control force and moment. $u, v, w$ are velocity components, $\mathbf{v}_{0}=(u, v, w), p, q, r$ are roll, pitch and yaw, $\mathbf{\Omega}=(p, q, r) . \psi$ is velosity potential[19].

\subsection{Kinematics of airship}

The kinematics equations of airship are:

$$
\left[\begin{array}{c}
\dot{\varphi} \\
\dot{\vartheta} \\
\dot{\psi}
\end{array}\right]=\left[\begin{array}{ccc}
1 & \sin \varphi \tan \vartheta & \cos \varphi \tan \vartheta \\
0 & \cos \varphi & -\sin \varphi \\
0 & \frac{\sin \varphi}{\cos \vartheta} & \frac{\cos \varphi}{\sin \vartheta}
\end{array}\right]\left[\begin{array}{l}
p \\
q \\
r
\end{array}\right]
$$

$$
\left[\begin{array}{c}
\dot{x} \\
\dot{y} \\
\dot{z}
\end{array}\right]=\left[\begin{array}{ccc}
\cos \psi \cos \vartheta & \sin \psi \cos \vartheta & -\sin \vartheta \\
\cos \psi \sin \vartheta \sin \varphi-\sin \psi \cos \varphi & \sin \psi \sin \vartheta \sin \varphi+\cos \psi \cos \varphi & \cos \vartheta \sin \varphi \\
\cos \psi \sin \vartheta \cos \varphi+\sin \psi \sin \varphi & \sin \psi \sin \vartheta \cos \varphi-\cos \psi \sin \varphi & \cos \vartheta \cos \varphi
\end{array}\right]^{T}\left[\begin{array}{c}
u \\
v \\
w
\end{array}\right]^{2}
$$

where, $\vartheta, \psi, \varphi$ are the pitch, yaw and roll angle respectively; $x, y, z$ are the global position coordinates respectively.

\section{Backstepping control design and error analysis}

Several places in this paper refer to filtering of a signal $x_{c}^{0}$ to produce a signal $x_{c}$ and its derivative ${ }^{\dot{x}_{c}}$. This is referred to as command filtering. The state space implementation of such a filter is[20]

$$
\begin{aligned}
& \dot{x}_{1}=x_{2} \\
& \dot{x}_{2}=-2 \zeta w_{n} x_{2}-w_{n}^{2}\left(x_{1}-x_{c}^{0}\right)
\end{aligned}
$$

where $x_{c}=x_{1}$ and $\dot{x}_{c}=x_{2}$. Note that if $x_{c}^{0}$ is bounded, then $x_{c}$ and $\dot{x}_{c}$ are bounded and continuous. The transfer function from $x_{c}^{0}$ to $x_{c}$ is

$$
\frac{X_{c}(s)}{X_{c}^{0}(s)}=H(s)=\frac{w_{n}^{2}}{s^{2}+2 \zeta w_{n} s+w_{n}^{2}}
$$

which has a unity gain at low frequencies, damping ratio $\zeta$ and undamped nature frequency $w_{n}$. The error $\left|x_{c}^{0}(t)-x_{c}(t)\right|$ is small if the bandwidth of $X_{c}^{0}(s)$ is less than the bandwidth of $H(s)$. If the bandwidth of $x_{c}^{0}$ is known and the goal of the filter is to generate $x_{c}$ and its derivative with $\left|x_{c}^{0}-x_{c}\right|$ small, then the designer simply chooses ${ }^{w_{n}}$ sufficiently large.

It is to be noted that, the yaw control system of the flexible airship can be obtained as the second-order form from equations (1) and (4),

$$
\left\{\begin{aligned}
\dot{\psi}= & \frac{1}{\cos \vartheta} r \\
\dot{r}=\frac{1}{\mathbf{M}_{22}(3,3)}\left[-0+\mathbf{M}_{\mathbf{1}}(1,3)+\mathbf{M}_{\mathbf{G}}(1,3)+M_{A S}(1,3)+\mathbf{M}_{\mathbf{A D}}(1,3)+\mathbf{M}_{\mathbf{C}}(1,3)\right. & \left.-\mathbf{M}_{21}(3,1: 3) \dot{\mathbf{v}}_{0}-\mathbf{M}_{23} \ddot{\mathbf{q}}(1: 4,1)\right]
\end{aligned}\right.
$$

where, $\mathbf{M}_{\mathbf{C}}(1,3)=k \delta_{r},{ }^{k}$ is rudder coefficient.

Let $x_{1}=\psi, x_{2}=r, u_{\text {input }}=\delta_{r}$. Then we analyze the second-order state-space equations as follow

$$
\left\{\begin{array}{l}
\dot{x}_{1}=G_{1} x_{2} \\
\dot{x}_{2}=f_{2}+G_{2} u_{\text {input }}
\end{array}\right.
$$

where the

coefficients $G_{i}(i=1,2)$ and $f_{2}$ are $G_{1}=\frac{1}{\cos \vartheta}$,

$$
\begin{aligned}
G_{2}= & \frac{1}{\mathbf{M}_{22}(3,3)} k, \\
f_{1}= & \frac{1}{\mathbf{M}_{22}(3,3)}\left[-0+\mathbf{M}_{\mathbf{I}}(1,3)+\mathbf{M}_{\mathbf{G}}(1,3)+M_{A S}(1,3)\right. \\
& \left.+\mathbf{M}_{\mathbf{A D}}(1,3)-\mathbf{M}_{21}(3,1: 3) \dot{\mathbf{v}}_{\mathbf{0}}-\mathbf{M}_{23} \ddot{\mathbf{q}}(1: 4,1)\right]
\end{aligned}
$$


Considering the virtual system

$$
\dot{x}_{1}=G_{1} v
$$

$v$ is the virtual term, and defining the position tracking error

$$
z_{1}=x_{1 d}-x_{1}
$$

where $x_{1 d}$ is the reference state.

And the Lyapunov function

$$
V_{1}=\frac{1}{2} z_{1}^{T} z_{1}
$$

whose dynamics can be derived as

$$
\dot{V}_{1}=z_{1}^{T} \dot{z}_{1}=z_{1}^{T}\left(\dot{x}_{1 d}-\dot{x}_{1}\right)=z_{1}^{T}\left(\dot{x}_{1 d}-G_{1} \boldsymbol{v}\right)
$$

To drive the tracking error to zero asymptotically, the traditional choice is to pick

$$
v=G_{1}^{-1}\left(\dot{x}_{1 d}+k_{1} z_{1}\right)
$$

In order to introduce the integral action, we choose the behavior of the virtual control as

$$
v=G_{1}^{-1}\left(\dot{x}_{1 d}+k_{1} z_{1}+\lambda \xi\right)
$$

where $k_{1}$ and $\lambda$ are positive constants at the disposal of the designer and

$\xi=\int_{0}^{t} z_{1}(\tau) d \tau$ is the integral action of the tracking error.

By introducing the integral action into the stabilizing function, we can make sure that the tracking error is converged to zero at the steady state despite the presence of the disturbance and inaccuracy, just like the influence of flexible model.

Then consider the tracking error

$$
\begin{gathered}
z_{2}=v-x_{2}=G_{1}^{-1}\left(\dot{x}_{1 d}+k_{1} z_{1}+\lambda \xi-x_{2}\right) \\
\text { Hence } \dot{z}_{1}=G_{1} z_{2}-k_{1} z_{1}-\lambda \xi \\
\dot{z}_{2}=G_{1}^{-1}\left(\ddot{x}_{1 d}+k_{1} \dot{z}_{1}+\lambda \xi\right)-\left(f_{2}+G_{2} u_{\text {input }}\right)
\end{gathered}
$$

The control Lyapunov function

$$
V_{2}=\frac{\lambda}{2} \xi^{T} \xi+\frac{1}{2} z_{1}^{T} z_{1}+\frac{1}{2} z_{2}^{T} z_{2}
$$

The derivative with respect to time of equation (19) reads

$$
\begin{aligned}
\dot{V}_{2} & =\lambda \xi^{T} \dot{\xi}+z_{1}^{T} \dot{z}_{1}+z_{2}^{T} \dot{z}_{2} \\
& =-k_{1} z_{1}^{T} z_{1}+z_{2}^{T}\left(\left(G_{1}^{T}+\lambda G_{1}^{-1}-k_{1}^{2} G_{1}^{-1}\right) z_{1}+k_{1} z_{2}-k_{1} \lambda G_{1}^{-1} \xi+G_{1}^{-1} \ddot{x}_{1 d}-f_{2}-G_{2} u_{\text {input }}\right)
\end{aligned}
$$

In order to render the non-positivity of the control Lyapunov derivative function, we choose the input as
Then $\dot{V}_{2}=-k_{1} z_{1}^{T} z_{1}-k_{1} z_{2}^{T} z_{2} \leq 0$.

\section{Simulation results}

Table 1. Parameters of model.

\begin{tabular}{|c|c|c|c|}
\hline$m(\mathrm{~kg})$ & 2682.1 & $I_{x}\left(\mathrm{~kg} \cdot \mathrm{m}^{2}\right)$ & 108340 \\
\hline$I_{y}\left(\mathrm{~kg} \cdot \mathrm{m}^{2}\right)$ & 288190 & $I_{z}\left(\mathrm{~kg} \cdot \mathrm{m}^{2}\right)$ & 217220 \\
\hline$m_{11}(\mathrm{~kg})$ & 505.7455 & $m_{22}(\mathrm{~kg})$ & 5073.7 \\
\hline$m_{33}(\mathrm{~kg})$ & 5073.7 & $m_{44}(\mathrm{~kg})$ & 68879 \\
\hline$m_{55}(\mathrm{~kg})$ & 585400 & $m_{66}(\mathrm{~kg})$ & 585400 \\
\hline$L(m)$ & 50 & $\begin{array}{l}\left(x_{G}, y_{G}, z_{G}\right) \\
(m)\end{array}$ & $(0,0,3.605)$ \\
\hline
\end{tabular}

The following controller parameters are used: $k_{1}=0.1, k_{2}=0.1$, and $\lambda=0.01$; the initial conditions are: $\psi=0$ an $\delta_{r}=0$. The reference trajectory chosen for $\psi_{d}$ is:

$$
\psi_{d}=\left\{\begin{array}{rc}
\left(1-e^{-0.05 t}\right) \cdot \frac{\pi}{2} & 0 \leq t<100 \\
\frac{\pi}{2}+\left(1-e^{-0.05(t-100)}\right) \cdot \frac{\pi}{6} & 100 \leq t<200 \\
\frac{\pi}{2}+\frac{\pi}{6}-\left(1-e^{-0.05(t-200)}\right) \cdot \frac{\pi}{3} & 200 \leq t \leq 400
\end{array} .\right.
$$

In order to close to practical applications, the following limitations in magnitude and rate of change of actuator (rudder angle) have also been considered in the simulation model.

$$
F_{T}=270 \mathrm{~N},\left|\delta_{r}\right| \leq 30 \mathrm{deg},\left|\dot{\delta}_{r}\right| \leq 5 \mathrm{deg} / \mathrm{s} \text {. }
$$

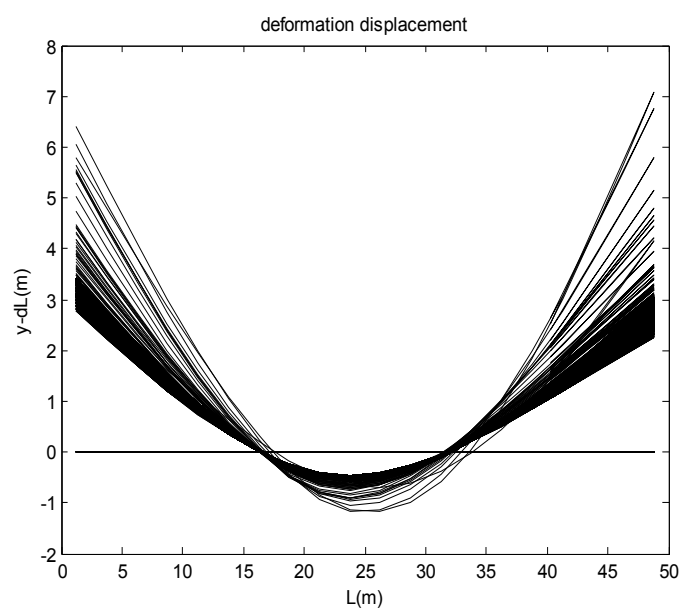

$u_{\text {input }}=G_{2}^{-1}\left(\left(G_{1}^{T}+\lambda G_{1}^{-1}-k_{1}^{2} G_{1}^{-1}\right) z_{1}+2 k_{1} z_{2}-k_{1} \lambda G_{1}^{-1} \xi+G_{1}^{-1} \ddot{x}_{1 d}-f_{2}\right)$ Figure 3. Airship deformation in Y-axis $\left({ }^{\delta_{r}}=30^{\circ}\right)$. 
Figure 3 shows the results of the flexible airship in the open-loop conditions. Rudder was set $\delta_{r}=30^{\circ}$ and airship kept turning circle with the speed $u=8.7 \mathrm{~m} / \mathrm{s}$. From the results, we can clearly see the deformation of the airship in Y-axis due to the force and moment of the rudder. Compared to the middle of the airship, the nose and the rear of the airship have larger deformation, about $6 \mathrm{~m}$ and $7 \mathrm{~m}$ respectively in max. When the airship turned circle with constant radius, the deformation of the nose and rear kept at about $3 \mathrm{~m}$.

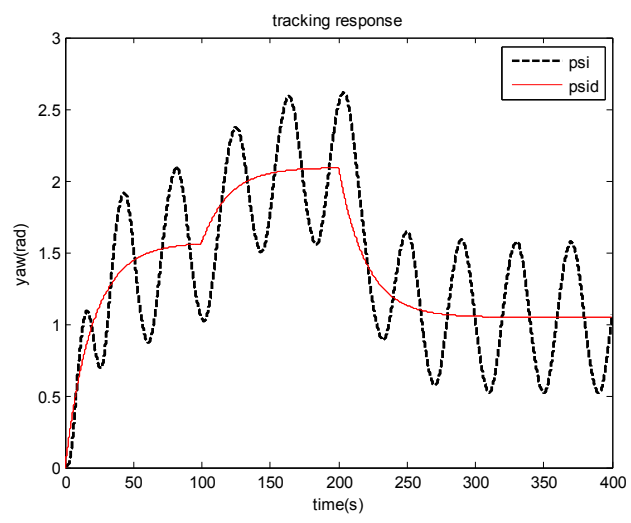

Figure 4. Time evolution of yaw performance without the integral action (solid is reference and dotted is tracking response).

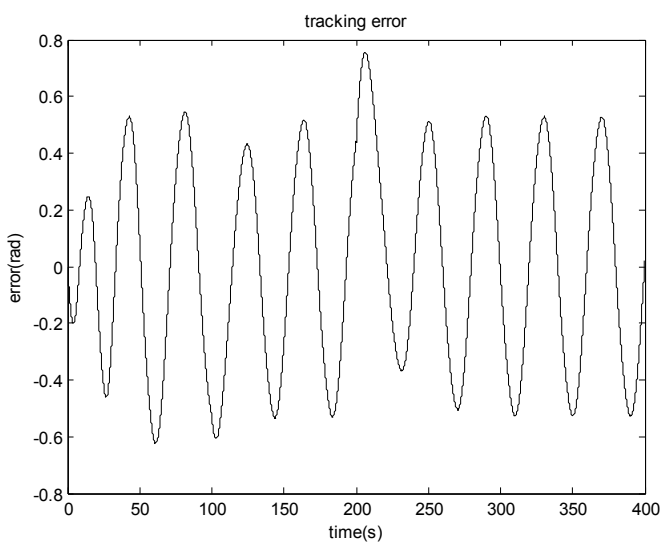

Figure 5. Tracking error without integral action.

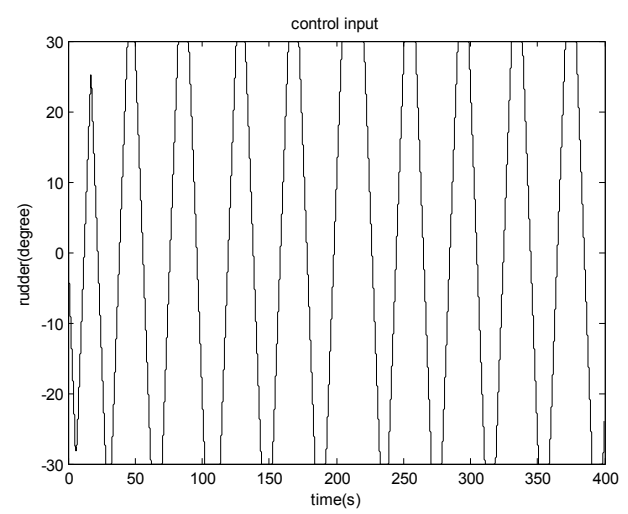

Figure 6. Control input without integral action.
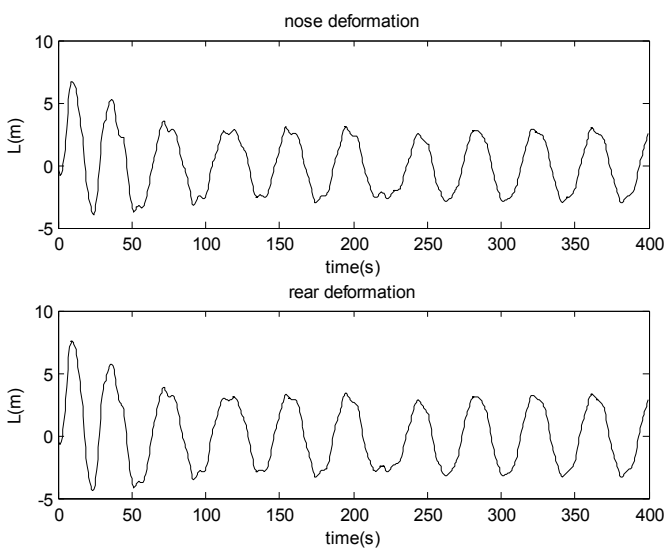

Figure 7. Deformation in $\mathrm{Y}$-axis (no integral action).

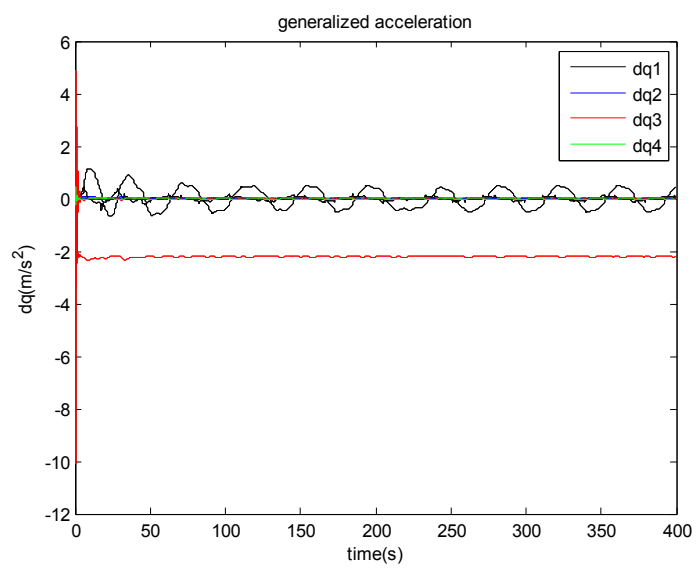

Figure 8. Time evolution of generalized acceleration (without integral action).

Our proposed integral command filtered backstepping nonlinear controller for flexible system is based on the Lyapunov function design methodology and essentially has nonlinear control properties. Because the system can be proven stable by the Lyapunov function through energy consumption, the backstepping controller with integral section has much higher stability range than the conventional controller (without integral section)

The reference signal is trapezoidal input in order to close to the practical applications and slightly smoothed to avoid discontinuities that would demand instantaneous changes in the control signals.

From our control design, we can see that the integral action can be easily removed from the backstepping controller by setting $\lambda=0$. Figs 4 to 8 are the simulation results for the backstepping without the integral action. At time $\mathrm{t}=100$, reference is commanded from $\mathrm{pi} / 2 \mathrm{rad}$ to $2 \mathrm{pi} / 3 \mathrm{rad}$; then at time $\mathrm{t}=200 \mathrm{~s}$, reference is decreased with a magnitude $\mathrm{pi} / 3 \mathrm{rad}$. From the tracking error plot in Fig.5, we can see that there is a dynamic error of about 0.6 radians. This is because of the bandwidth limitation of the conventional backstepping controller, which can be seen from control input (Fig.6). The rudder keeps saturating due to the rate limitation of the actuator. So the tracking error vibrates near the equilibrium point. During 
the tracking process, the deformation of airship is fluctuating due to the control input (see Fig.7), which may influence the accurate flight control.

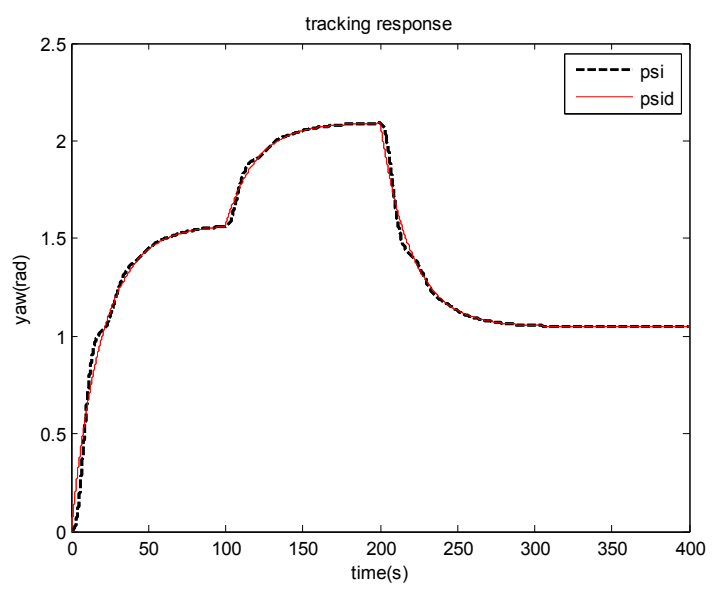

Figure 9. Time evolution of yaw performance with integral action (solid is reference and dotted is tracking response).

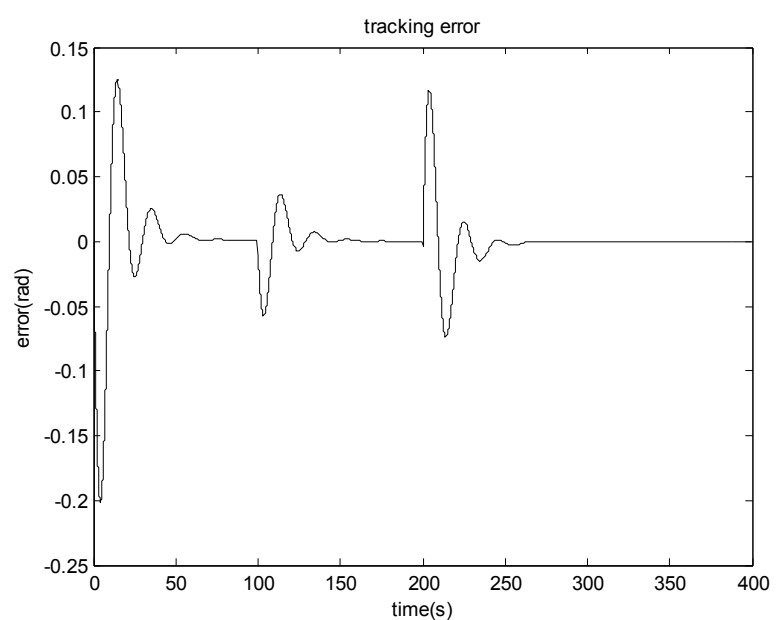

Figure 10. Tracking error of the backstepping controller with the integral action.

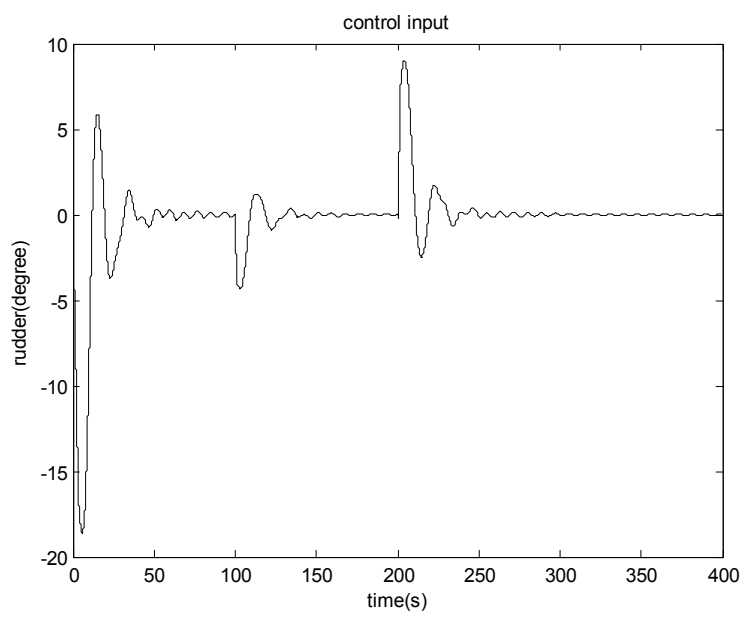

Figure 11. Control input with integral action.
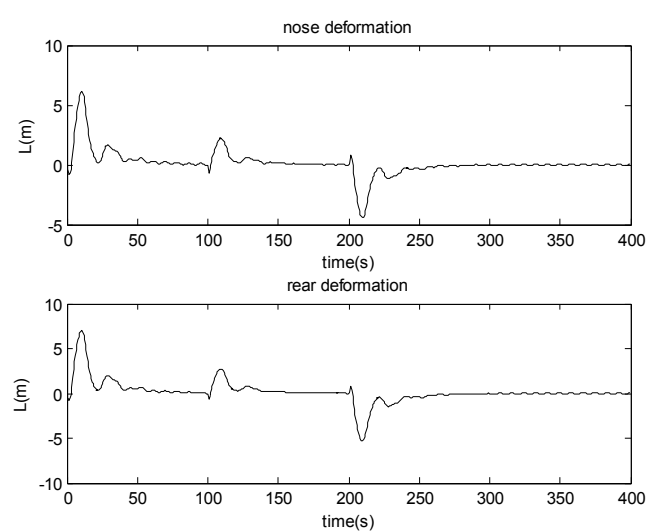

Figure 12. Airship deformation in $\mathrm{Y}$-axis (with integral action).

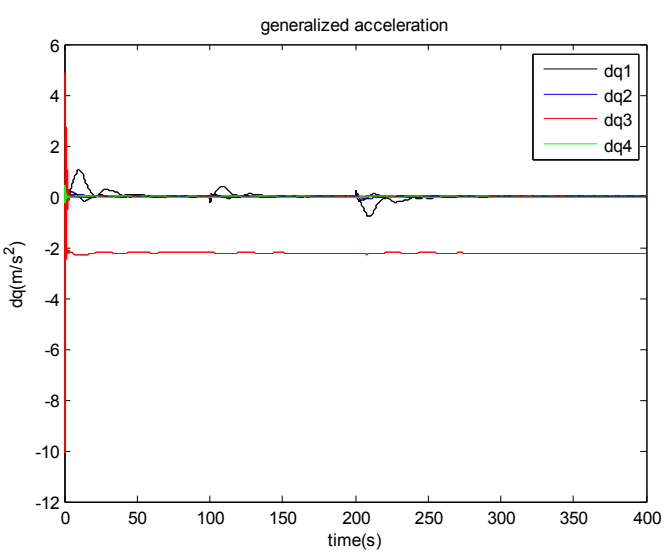

Figure 13. Time evolution of generalized acceleration (with integral action).

The same command position reference is used for the integral backstepping nonlinear controller. The performance is shown from Fig.9 to fig.13. With the backstepping nonlinear controller added integral action, we can obtain that the tracking error has been greatly reduced to below 0.01 radians during the steady process as can been seen in Fig.10. Even at the steep changes, the tracking error is only about 0.13 radians, which is much smaller than that of the conventional controller. The fast tracking performance is preserved. Fig. 9 shows a better match to the reference, in comparison with Fig. 4. Comparing Fig. 11 with Fig.6, the rudder response is rather smooth when moderate changes in yaw angle are demanded. What is more, with the integral action, the backstepping controller is adapted to the bandwidth limitation of the rate of actuators and makes the simulation more close to reality. From Fig. 12 we can see that the deformation tendency of airship just opposite to the rudder (control input), and rapidly converge to zero, which is further improvement than that of Fig.7. Fig.13 shows that the integral backstepping controller considering flexible factors could resist the flexible uncertainty and converge the generalized acceleration to steady values. All of these above prove that integral backstepping control with flexible factors considered is more adapted to limitation of actuators and shows better performance to control the system against the flexible uncertainty presented in the motion control systems. 


\section{Conclusion}

In this paper, the dynamic modeling of the flexible airship has been presented, which offers the precise model for control near the truth. And then it has shown the process for the design of a nonlinear controller employing Lyapunov and command filtered Backstepping techniques to control the yaw angle of the flexible system. In order to make up the flexible uncertainty factor and the limitation of the rate of actuators, integral action is considered in the design controller. Numerical simulations are performed to verify the performance of the proposed control law. The results are shown that the proposed integral command filtered backstepping control algorithm could stabilize the flexible system and drive the flexible airship to the desired trajectory of yaw angle.

\section{References}

1. Oh S, Kang S, Lee $\mathrm{K}$, et al. Flying display: autonomous blimp with real-time visual tracking and image projection[C]//Intelligent Robots and Systems, 2006 IEEE/RSJ International Conference on. IEEE, 2006: 131-136.

2. Tischler, M.B., Ringland, R.R., and Jex, H.R., "Heavy Airship Dynamics," Journal of Aircraft, Vol.20, No.5, 1983, pp.425433.doi: $10.2514 / 3.44888$

3. Jex, H.R., and Gelhausen, P., "Pre- and Post-FlightTest Models Versus Measured Skyship-500 Control Responses," 7th AIAA Lighter-Than-Air Technology Conference, AIAA, NewYork, 1987, pp.87-97.

4. Amann, J.H., “ A Comparison of a Nonlinear Flight Dynamic Simulation of an Airship with Flight Test Results," 7th AIAA Lighter-Than-Air Technology Conference, AIAA, NewYork, 1987, pp.78-86.

5. Cook, M.V., Lipscombe, J.M., and Goineau, F., "Analysis of the Stability Modes of the Non-Rigid Airship," The Aeronautical Journal, Vol.104, No.1036, 2000, pp.279-290.

6. Schmidt, D.K., "Modeling and Near-Space Stationkeeping Control of a Large High-Altitude Airship," Journal of Guidance, Control, and Dynamics,Vol.30,No.2,2007,pp.540-547.

7. Li, Y., and Nahon, M., "Modeling and Simulation of Airship Dynamics," Journal of Guidance, Control, and Dynamics, Vol.30, No.6,2007, pp.1691-1700.
8. Calise A., Nakwan K., 2002 " Adaptive compensation for flexible dynamics". AIAA Guidance, navigation and control conference. Montery, Canada.

9. Bianchin M., Quaranta G., Mantegazza P., 2003 "State space reduced order models for static aero elasticity and flight mechanics of flexible aircrafts". 17 national conference AIDAA, Italy,.

10. McLean, D.. Automatic Flight Control Systems. Prentice Hall. 1990

11. Nichols, R.A., Reichert, R.T., and Rugh, W.J.. Gain scheduling for controllers: A flight control example. IEEE Trans. Contr. Syst. Tech., 1993,1(2): 69-78.

12. Ochi, Y. and Kanai, K.. Design of restructurable flight control systems using feedback linearization. Journal of Guidance, Control, and Dynamics, 1991,14(5),903-911.

13. M. Krstic, I. Kanellakopoulos, and P. V. Kokotovic, Nonlinear and Adaptive Control Design, John Wiley Sons, Inc, New York, 1995.

14. E. Freund and R. May, "Nonlinear Path Control in Automated Vehicle Guidance," IEEE Transactions on Robotics and Automation, 1997,13(1):pp.4940.

15. R. Fierro and F. L. Lewis, "Control of a nonholonomic mobile robot: Backstepping kinematics into dynamics," in Proceedings of CDC'95, 34th IEEE Conference on Decision and Control, New Orleans, LA, USA, Dec. 1995, pp. 3805-3810.

16. P. Encarnacao and A. Pascoal, "Combined trajectory tracking and path following for marine craft," 40th IEEE Conference on Decision and Control, Mar. 2001.

17. Härkegård, O., "Backstepping and Control Allocation with Applications to Flight Control," Ph.D. Thesis, Linköping Univ., Linköping, Sweden,2003.

18. S.P.Jones, J.D.Delaurier, Aerodynamic estimation techniques for aerostats and airships, Journal of aircraft, VoL.20 No.2,1983.

19. Yuwen Li, Dynamics Modeling and Simulation of Flexible Airships, Doctor thesis, 2008.

20. Han D, Wang X, Chen L, et al. Command-filtered backstepping control for a multi-vectored thrust stratospheric airship [J]. Transactions of the Institute of Measurement and Control, 2015 